\title{
Article
}

\section{On-Surface Synthesis of Boroxine-Based Molecules}

\author{
Elia Turco ${ }^{1}$, Matus Stredansky ${ }^{1,2} \mathbb{D}$, Roberto Costantini ${ }^{2}{ }^{4}$, Javier A. Martinez ${ }^{3}$, Martina Dell'Angela ${ }^{2} \mathbb{D}$, \\ Elena Zerbato ${ }^{4}$, Daniele Toffoli ${ }^{4}{ }^{(}$, Giovanna Fronzoni ${ }^{4}$, Alberto Morgante ${ }^{1,2}$, Luca Floreano ${ }^{2}(\mathbb{B}$ \\ and Albano Cossaro ${ }^{2,4, *}$ (1)
}

1 Department of Physics, University of Trieste, 34127 Trieste, Italy; eliaturco@gmail.com (E.T.); mstredansky@fhi-berlin.mpg.de (M.S.); morgante@iom.cnr.it (A.M.)

2 CNR-IOM, Istituto officina dei Materiali, 34149 Trieste, Italy; costantini@iom.cnr.it (R.C.); dellangela@iom.cnr.it (M.D.); floreano@iom.cnr.it (L.F.)

3 Institute of Materials Science and Technology (IMRE), University of Havana, Havana 10400, Cuba; javmar@imre.uh.cu

4 Department of Chemical and Pharmaceutical Sciences, University of Trieste, 34127 Trieste, Italy; ELENA.ZERBATO@studenti.units.it (E.Z.); toffoli@units.it (D.T.); fronzoni@units.it (G.F.)

* Correspondence: acossaro@units.it

check for

updates

Citation: Turco, E.; Stredansky, M.; Costantini, R.; Martinez, J.A.; Dell'Angela, M.; Zerbato, E.; Toffoli, D.; Fronzoni, G.; Morgante, A.;

Floreano, L.; et al. On-Surface Synthesis of Boroxine-Based Molecules. Chemistry 2021, 3, 1401-1410. https://doi.org/10.3390/ chemistry3040101

Academic Editor: Roland C. Fischer

Received: 18 October 2021

Accepted: 24 November 2021

Published: 30 November 2021

Publisher's Note: MDPI stays neutral with regard to jurisdictional claims in published maps and institutional affiliations.

Copyright: (C) 2021 by the authors. Licensee MDPI, Basel, Switzerland. This article is an open access article distributed under the terms and conditions of the Creative Commons Attribution (CC BY) license (https:// creativecommons.org/licenses/by/ $4.0 /)$.

\begin{abstract}
The on-surface synthesis of boroxine-containing molecules can be a convenient method of introducing specific functionalities. Here, we show the validity of a previously described synthesis protocol on the $\mathrm{Au}$ (111) surface by applying it to a different molecular precursor. We study in detail the assembly of the precursor, highlighting possible intermediate stages of the condensation process. We combine scanning tunneling microscopy and X-ray spectroscopies to fully characterize both the morphology and the electronic properties of the system. DFT calculations are presented to assign the main electronic transitions originating the B K-edge absorption spectrum. The study paves the way to a facile strategy for functionalizing a surface with molecules of tailored sizes and compositions.
\end{abstract}

Keywords: boroxine; on-surface synthesis; NEXAFS; STM; functionalization; DFT calculations

\section{Introduction}

On-surface synthesis of 2D organic structures is a convenient bottom-up approach for tailoring both the chemistry and the morphology of surfaces and for the synthesis of complex hybrid interfaces of potential interest in the development of organic based devices [1-5]. Of the different synthetic routes reported to date, the condensation of boronic precursors is one of the most notable because of its facile protocol as well as the robustness of the resulting structures. Boronic condensation was the first [6] synthetic method for the formation of 3D covalent organic frameworks (COFs) [7-9], and it is still widely used. Self-condensation of boronic acids can lead to the formation of boroxine, a six-membered ring of alternating oxygen and boron atoms. Alternatively, it can lead to condensation of boronic acids with diols form boronate groups [7]. In recent years, the robustness and nano-sized arrangement of boronic COFs have encouraged research into transposing their synthesis on surfaces. Single-layered 2D COFs have been successfully grown on surfaces and fully characterized in terms of their morphology [10-14]. Their use as templates has been demonstrated in a shape-matching guest-host approach [15]. Whereas the condensation of molecules with two boronic groups allows for obtaining extended 2D structures, the use of single-terminated precursors leads to the formation of boroxine-containing molecular moieties. We recently explored the latter possibility with the synthesis of triphenyl boroxine molecule on the $\mathrm{Au}(111)$ surface, which was obtained by sublimation of the phenyl boronic acid precursor under ultra-high vacuum conditions [16]. We have shown that the interaction between boroxine and $\mathrm{Au}(111)$ surfaces is characterized by the presence of interface electronic states, which represent preferential channels for ultrafast charge delocalization. These findings suggest the employment of boroxine molecules 
as platforms for the hosting of guest molecules is able to mediate charge transport at the interface between organic films and metal substrates. Therefore, by changing the structure of the boronic precursor, the size and the composition of the resulting macromolecule can be tailored in order to promote a specific recognition process. It is therefore important to verify the possibility of extending the boroxination reaction to other mono-boronic species and to better characterize the on-surface condensation process. Here, we investigate the condensation of naphthyl-boronic acid (NBA) on the $\mathrm{Au}(111)$ surface aiming to achieve the on-surface synthesis of the tri-naphthyl boroxine (TNB). The condensation scheme as well as a sketch of the molecules involved is represented in Figure 1.

NBA

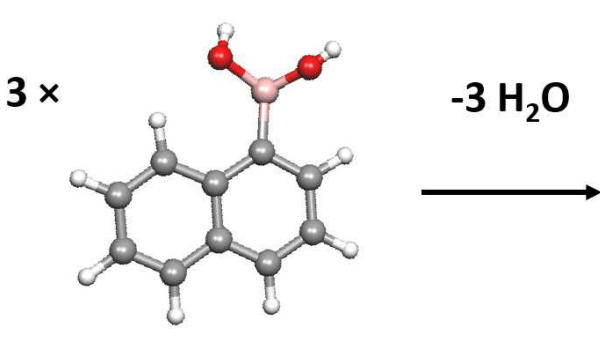

TNB

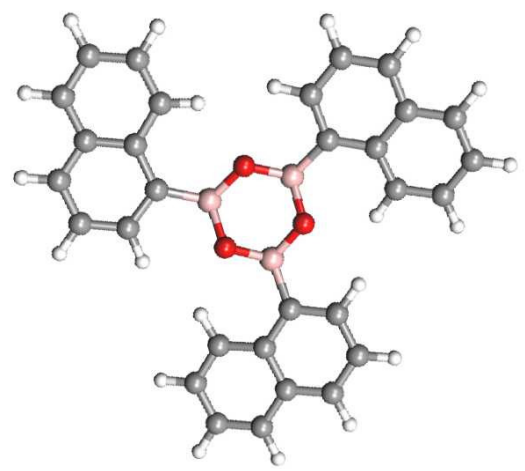

Figure 1. Condensation of three NBA molecules leads to the formation of a TNB molecule with release of three water molecules.

We performed a combined scanning tunneling microscopy (STM) and X-ray spectroscopy characterization of the NBA monolayers obtained by depositing the molecules, as kept at different temperatures $T_{s}$, on the clean $\mathrm{Au}(111)$ surface. We identified three phases, characterized by different domain symmetries and molecular moieties, which we named: (i) the low temperature (LT) phase, obtained for $T_{s} \approx 230-270 \mathrm{~K}$; (ii) the four-leaf (dimer pairing) phase, obtained for $T_{s} \approx 270-300 \mathrm{~K}$; and (iii) the trimer phase, obtained for $T_{s} \approx 330 \mathrm{~K}$. Phrase iii is characterized by the long-range ordering of TNB molecules.

\section{Materials and Methods}

$\mathrm{An} \mathrm{Au}(111)$ single crystal was cleaned by cycles of $\mathrm{Ar}^{+}$sputtering and annealing to $750 \mathrm{~K}$. NBA (Merck KGaA, Darmstadt, Germany, purity, >95\%) was deposited from a Knudsen cell at $330 \mathrm{~K}$. The growth of multilayered films was observed at sample temperature $T_{s} \lesssim 230 \mathrm{~K}$. The deposition time to obtain the sub-monolayer coverage of the films presented here was 4-5 min with the evaporator $15 \mathrm{~cm}$ from the sample.

STM measurements were performed at the joint CNR-Elettra OSMOS laboratory in Trieste, Italy, that is equipped with an Aarhus 150 microscope. Topographic images were collected by a tungsten tip with the sample cooled at liquid nitrogen temperature. Imaging parameters are indicated in the caption of the figures in terms of the tunneling current $\left(I_{t}\right)$ and sample bias $\left(V_{b}\right)$.

X-ray spectroscopy measurements were performed at the ANCHOR-SUNDYN [17] endstation of the ALOISA beamline at the Elettra Synchrotron, Trieste. O1s and C1s X-ray photoelectron spectroscopy (XPS) spectra were obtained with photon energy of $650 \mathrm{eV}$ and an overall resolution of $0.25 \mathrm{eV}$ and $\mathrm{B} 1 \mathrm{~s}$ with $300 \mathrm{eV}$ photon energy and overall resolution of $0.2 \mathrm{eV}$. For each spectrum, the $\mathrm{Au} 4 \mathrm{f}_{7 / 2}$ was also measured as reference to calibrate the binding energy of the photoemission spectra [18]. Near-edge X-ray absorption fine structure (NEXAFS) spectra were acquired in Auger yield with a photon energy resolution of $80 \mathrm{meV}$ for C and B K-edges and of $140 \mathrm{meV}$ for O K-edge.

We adopted here the theoretical approach we successfully applied to a similar system in a previous study [16]. The ground-state equilibrium geometry of gas-phase TNB was 
computed at the density functional theory (DFT) [19] level, employing the hybrid B3LYP xc functional [20-22] and a basis set of Slater-type orbitals (STO) of triple-zeta plus polarization quality (TZP).

The NEXAFS spectrum at the B K-edge was computed at DFT level with the transition potential scheme (DFT-TP) in which half an electron was removed from the initial core orbital and all the orbitals were relaxed until self-consistency was achieved. The DFT-TP approach ensured that relaxation effects upon the core-hole formation were adequately described [23]. The DFT-TP calculations employed the PW86xPerdew (PW86) generalized gradient approximation (GGA) for the xc functional [24]. An even-tempered quadruplezeta basis set with three polarization and three diffuse functions (ET-QZ3P-3DIFFUSE) was employed for the core-excited B atom, while a frozen core TZP.1s basis set was employed for the other atoms, to ensure the correct localization of the B 1s half core-hole. DFT-TP excitation energies were shifted with respect to the calculated $\triangle S C F$ ionization potential (IP) value by an amount given by the energy difference $\varepsilon_{1 \mathrm{~s}}^{\mathrm{TP}}-\Delta \mathrm{SCF}(1 \mathrm{~s})$. In the $\Delta \mathrm{SCF}$ calculations, the energy of the $1 \mathrm{~s}^{-1}$ ionic state was calculated with a spin polarized scheme. All calculations were done using the Amsterdam density functional (ADF) quantum chemistry code $[25,26]$.

\section{Results and Discussion}

\subsection{STM}

Figure 2 presents images of the monolayer phase as grown at a low temperature (LT phase). The large-scale image (Figure 2a) evidences that the herringbone reconstruction of the $\mathrm{Au}(111)$ surface is still visible, indicating a poor interaction between molecules and substrate. The zoomed image in Figure $2 b$ evidences a homogeneous self-assembly, where single molecules may be easily identified by their almost rectangular appearance, characterized by a certain convexity. This asymmetry of the molecular image can be ascribed to the presence of the boronic group on one side of the naphthalene body. Examining the molecular assembly more closely(see Figure $2 b, c$ ) allows for the identification of a cluster of four molecules as the recurring motif of the large molecular patterns. A single tetramer can be viewed as the unit cell of a regular $2 \mathrm{D}$ lattice. In Figure $2 b, c$, green and blue tetragons indicate the unit cells of two adjacent domains, dislocated by the length of half a cell from each other. The proliferation of these dislocation defects prevents the formation of larger domains. The convexity of the single molecule images alternates within the tetramers, giving them a chiral character. This highlights the specific role the boronic termination is playing in the assembly of this phase, which leads to a non-random mutual orientation of adjacent molecules. In Figure 2c, we suggest two possible interacting schemes between dimers of NBAthat preserve the symmetry of the tetramers as observed in the images. The images do not allow for further speculation about the intermolecular bonding scheme and about the role of the boronic termination in the assembly.

The results do not indicate that the tetrameric structures constitute the precursor state of the second NBA phase, which is the four-leaf phase whose images are reported in Figure 3. By deposition of NBA at the intermediate sample temperature 270-300 K, we observe that molecules aggregate in a characteristic four-leaf chiral motif, which extends all over the surface, establishing a long-range ordered phase without affecting the substrate herringbone reconstruction. Both left- and right-handed structures were observed, assembled in domains with a defined chirality (see Supplementary Materials). Looking at the intramolecular contrast in Figure 3c, each single four-leaf cluster seems to be formed by two adjacent dimers. The dimer molecules are much closer than in the LT phase and clearly display a continuum of density of states. Such dimers form a rigid unit, whereas the pairing of two dimers giving rise to the four-leaf motif is more fragile, since unpaired dimers can be detected occasionally at domain walls and defects. The possible nature of the intermolecular interactions of these dimers, as well as XPS data, will be discussed in Section 3.2. 

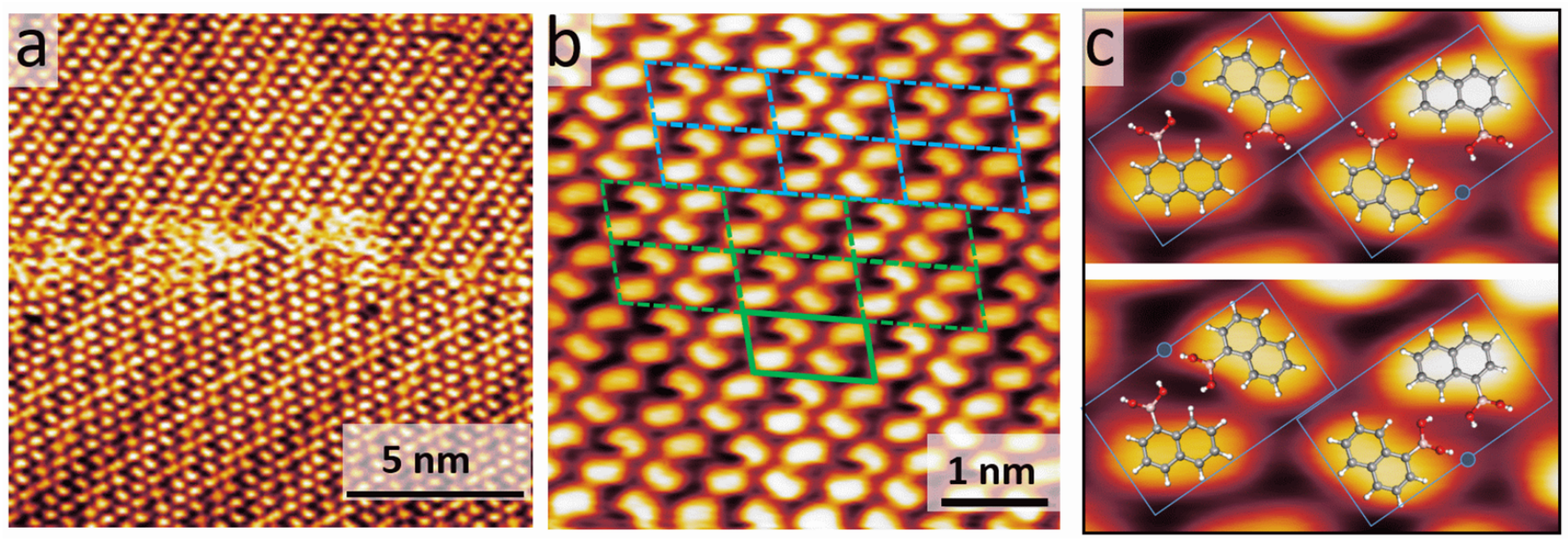

Figure 2. STM imaging of the LT phase. Unit cells of the assembly can be identified with the tetramers indicated by the green and blue lines (panel b). Two possible interacting schemes between dimers of NBA molecules superimposed to a tetramer are suggested (panel c). Measurement parameters: (a) $I_{t}=0.2 \mathrm{nA}, V_{b}=-0.91 \mathrm{~V}$; (b) $I_{t}=0.2 \mathrm{nA}, V_{b}=1.24 \mathrm{~V}$; (c) $I_{t}=0.2 \mathrm{nA}$, $V_{b}=-0.97 \mathrm{~V}$.
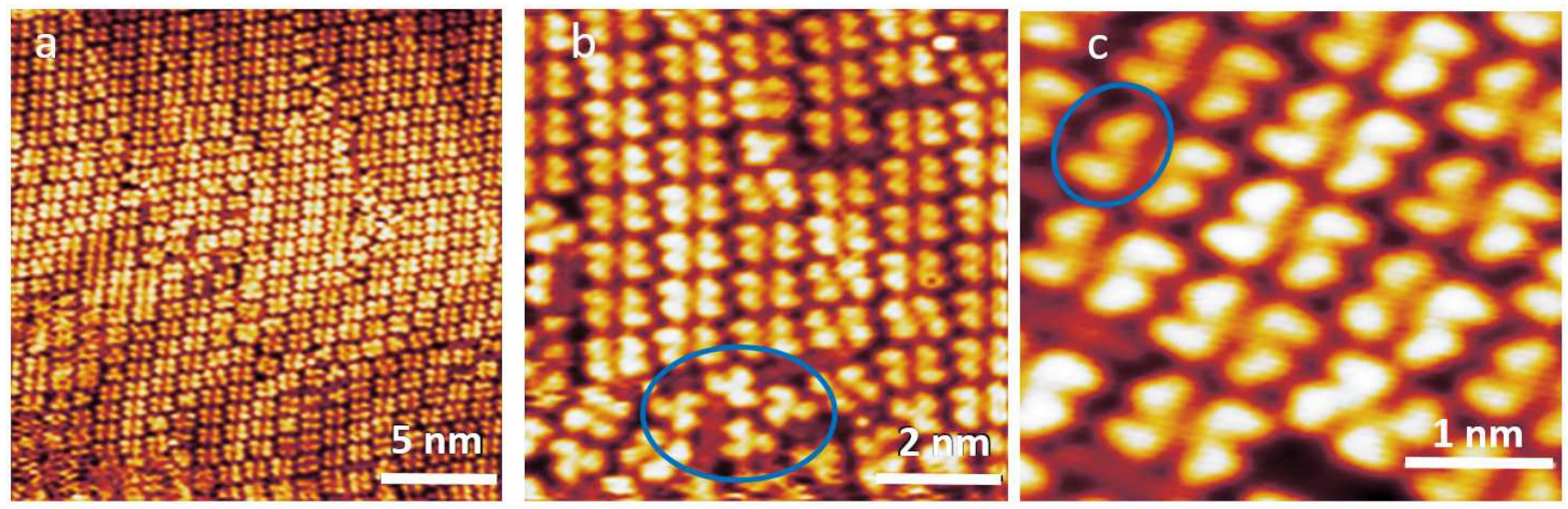

Figure 3. STM imaging of the four-leaf phase. (a) large scale image; (b) the blue circle evidencing the coexistence of TNB molecules; (c) The four-leaf structures can be viewed as 2 interacting dimers: the blue circle indicates one dimeric form. Measurement parameters: (a) $I_{t}=0.2 \mathrm{nA}, V_{b}=0.94 \mathrm{~V}$; (b) $I_{t}=0.2 \mathrm{nA}, V_{b}=0.94 \mathrm{~V}$; (c) $I_{t}=0.2 \mathrm{nA}, V_{b}=0.94 \mathrm{~V}$.

We recently reported that, in the case of phenyl boronic acid, the growth conditions used here to obtain the four-leaf (dimer) phase lead to the formation of covalent trimers, namely triphenylboroxine [16], upon boroxine condensation of the boronic terminations. Here, only a minority of the molecules undergo the condensation process with the formation of covalent trimers, at room temperature or lower, as circled in blue in Figure 3b. The complete boroxination of the NBA was observed in the trimer phase, obtained by the deposition at slightly higher temperatures of the sample $(330 \mathrm{~K})$ as shown in Figure 4 . We speculate that a higher temperature is needed for condensation of phenyl boronic acid because of the different molecular orientation in the condensation precursor stages and because of the different kinetics of the precursor molecules on the surface. The phenyl boronic acid was found to assemble at low temperatures in hydrogen bonded trimers, presenting the geometry of the condensed macromolecule [16]. In contrast, here, the substitution of the phenyl ring with a naphthyl group clearly affects the hydrogen bonding scheme and stabilizes the molecules at room temperature in the four-leaf geometry made of paired dimers (Figure 3), which does not promote trimer condensation. In addition, we verified that the annealing of both the LT and the four-leaf phase to $330 \mathrm{~K}$ does not lead to the trimer 
phase, but rather to a mixed phase of few trimers and four-leaf structures. Moreover, the overall molecular coverage decreases upon thermal treatment. In the specific case of the annealed four-leaf phase, this indicates that the desorption of the dimers competes with the formation of trimers. This may be because, starting from the four-leaf phase, a trimer can be formed only if a dimer is broken; this has a higher energetic cost than the desorption of the dimer. Conversely, during the deposition at $330 \mathrm{~K}$, the dimers that are synthesized may interact, while they are still trapped on the surface, with single NBA molecules provided by the ongoing deposition and condense into trimers. This mechanism is clearly affected by the kinetics of the precursors and may be the cause of the different condensation temperatures observed for naphthalene- and phenyl-based precursors. Further details about the evolution of the phases upon annealing are given in the Supplementary Materials.
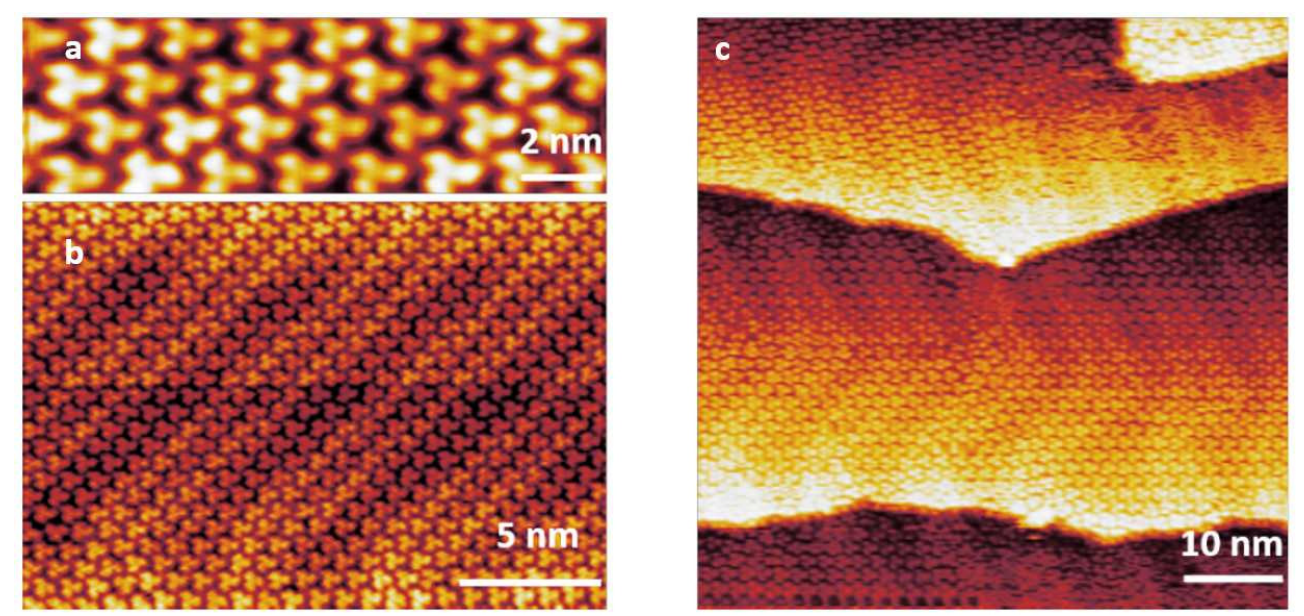

Figure 4. STM imaging of the trimer phase taken at different scales. Measurement parameters (a) $I_{t}=0.2 \mathrm{nA}, V_{b}=1.24 \mathrm{~V}$; (b) $I_{t}=0.2 \mathrm{nA}, V_{b}=1.24 \mathrm{~V}$; (c) $I_{t}=0.3 \mathrm{nA}, V b=1.39 \mathrm{~V}$.

\subsection{XPS}

To better characterize the condensation process imaged by STM we performed a XPS study of the four-leaf and of the trimer phases.

Figure 5 reports the O1s, C1s and B1s spectra taken in the two phases. The binding energies of the main peaks are consistent with the observations made on the triphenyl boroxine case [16] and, apart from a small shift toward lower energies in the O1s, are only poorly affected by the condensation process. Looking first at the O1s and $\mathrm{C} 1 \mathrm{~s}$ signals, the spectra were acquired at the same photon energy, thus making the $\mathrm{O} 1 \mathrm{~s} / \mathrm{C} 1 \mathrm{~s}$ ratio a good indicator of the stoichiometry of the two phases. The value, calculated upon fitting of the curves, indicates a 30\% relative drop in the O1s intensity. Assuming the NBA molecules are in their pristine form in the four-leaf phase, a 50\% drop would be expected, as the scheme in Figure 1 indicates. This suggests that in the four-leaf phase a partial condensation of the precursors had already taken place. A possible explanation, supporting both STM and XPS findings, is that dimers of molecules are formed upon condensation reaction and that the four-leaf clusters are the result of the interaction between two dimers. 


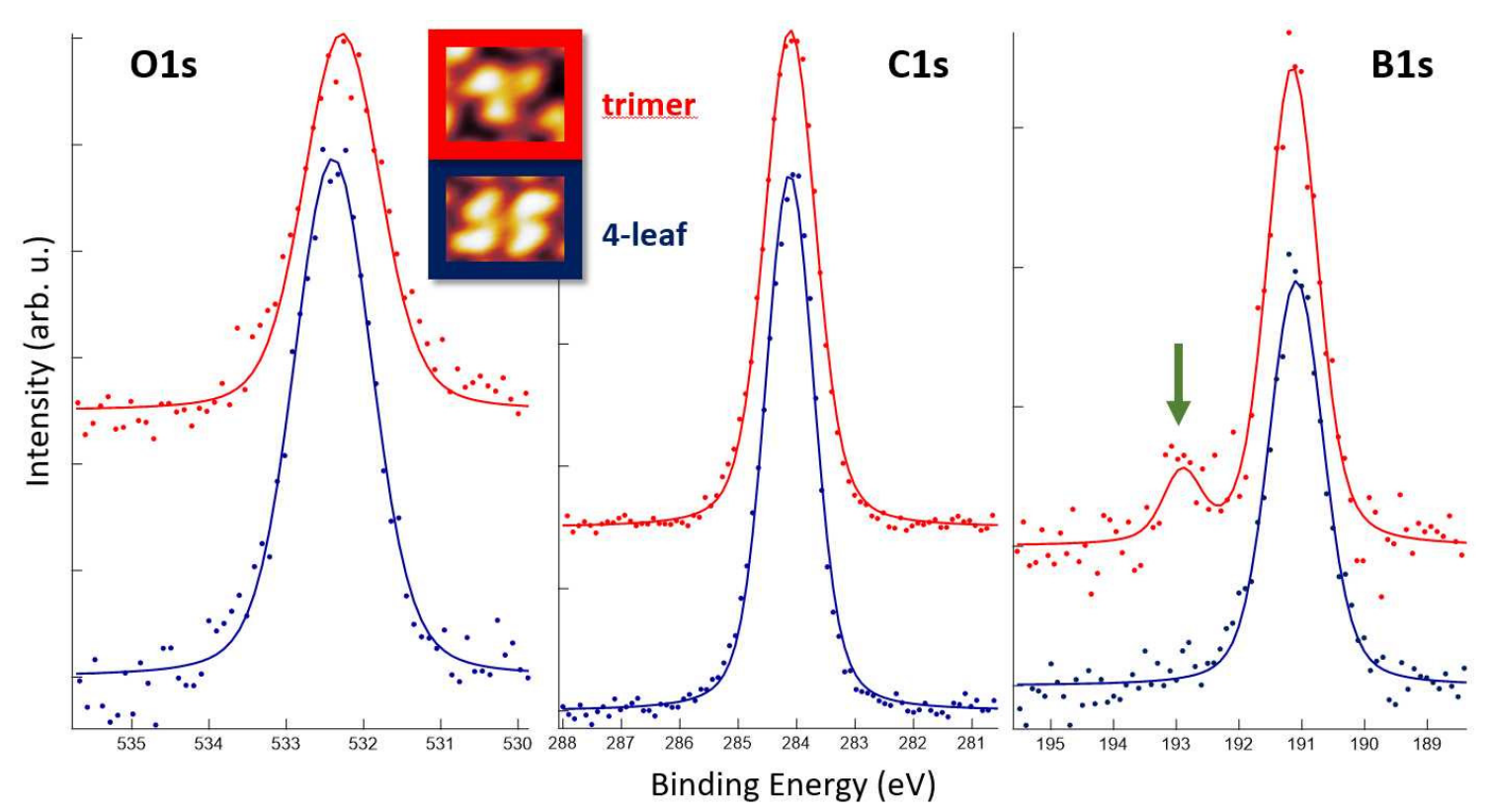

Figure 5. O1s, C1s and B1s photoemission spectra taken in the trimer (red markers) and four-leaf (blue markers) phase respectively, reported after integral background subtraction. Continue lines are the fitting curves.

Figure 6 reports a sketch of the possible dimer condensation process as well as the model we suggest to describe the structure of the clusters. A hydrogen bonding scheme involving the hydroxyl, indicated by dotted black lines in the figure, is possibly established between two adjacent dimers, similar to the interdimeric hydrogen bonds known to characterize the 3D assembly of boronic species $[27,28]$. This description of the four-leaf geometrical assembly needs to be confirmed by further investigation. In particular, we remark that the O1s/C1s intensity ratio measured upon different four-leaf phase preparations showed a certain variability. We believe that this is due to the metastable nature of the phase, characterized by the interaction between the precursor structures of the trimers. Small variations to the experimental conditions, such as sample temperature or NBA deposition rate, could favor the formation of TNB instead of the dimers coupling. As evidenced also in Figure 3b, a certain amount of TNB molecules is always present in the four-leaf phase, thus making the stoichiometry of the system variable if different preparations are compared.

$2 \times$

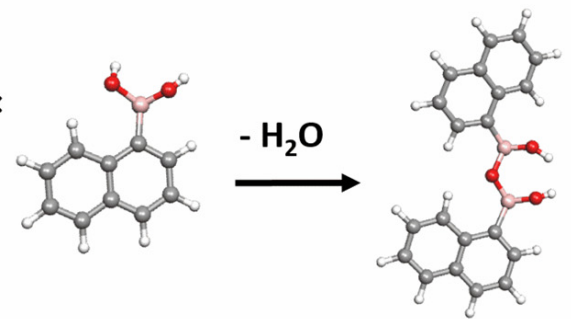

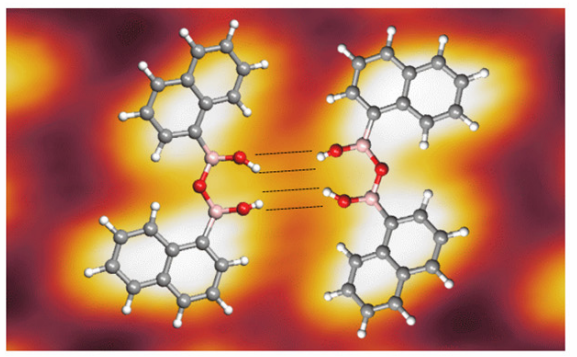

Figure 6. Proposed scheme for the dimer condensation (left) and superposition of the interacting dimers model (right) on the imaged four-leaf structure (image detail taken from Figure 3c).

The second notable aspect of the spectra in Figure 5 is the B1s lineshape. A novel, minor component is present on the trimer phase, as indicated by a green arrow in the figure. The nature of this component is not completely clear at the moment. Its binding energy, $\sim 193 \mathrm{eV}$, corresponds to the B1s of a 2D covalent framework of boroxine rings we recently synthesized on the $\mathrm{Au}(111)$ surface [29]. The boroxine rings were found to be interconnected by direct B-B bond. We speculate that here a small percentage of TNB molecules undergoes a further reaction, with the formation of larger molecules containing 
two boroxine rings linked through a B-B bond. This possibility will investigated further and will be discussed in a forthcoming publication.

\subsection{NEXAFS}

Figure 7 reports the $C$ and the B K-edge NEXAFS of the trimer phase, measured at two different angles between electric field polarization and surface normal, specifically $90^{\circ}$ (s pol) and $5^{\circ}$ (p pol). Both spectra present a strong dichroism, with signal corresponding to $1 s \rightarrow \pi^{*}$ transitions being maximum at $\mathrm{p} \mathrm{pol}$, indicating the flat adsorption geometry of TNB.
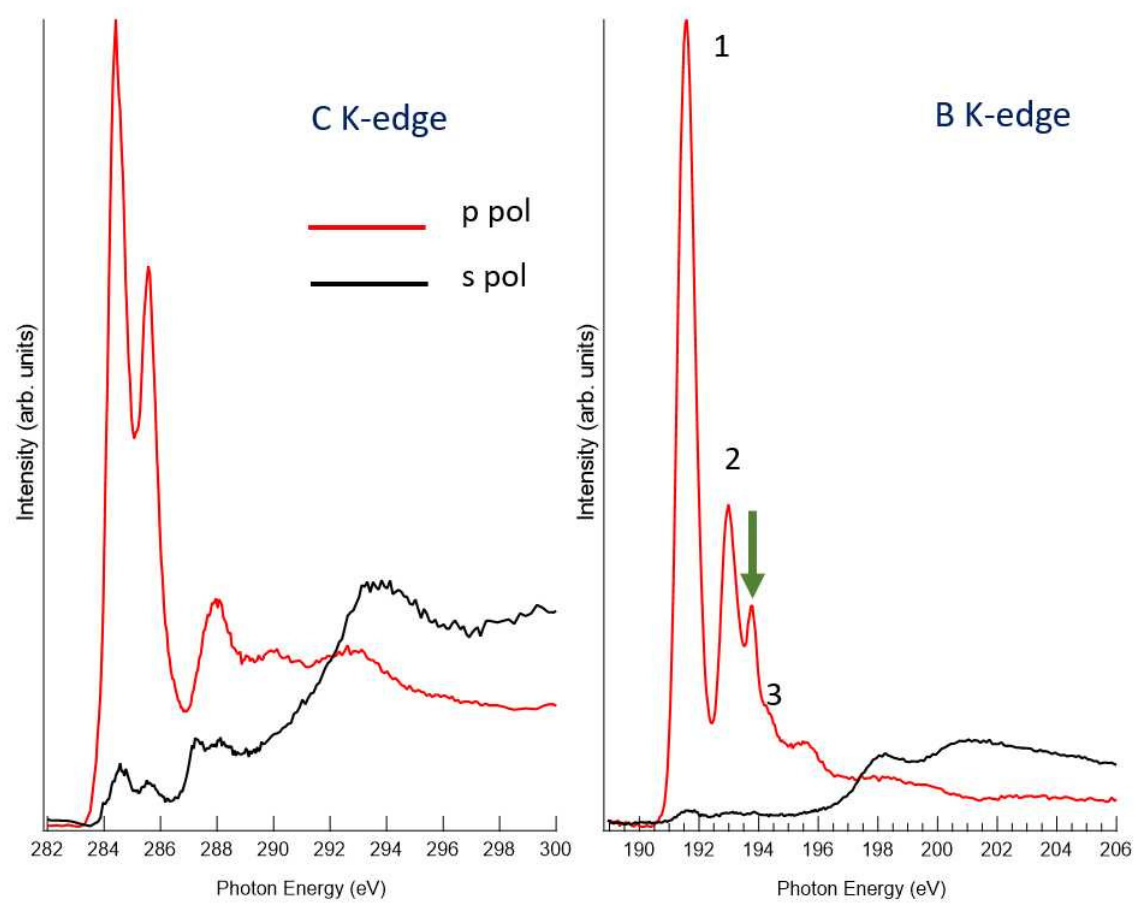

Figure 7. C and B K-edge NEXAFS of the trimer phase in $\mathrm{p}$ (red) and s (black) polarization. The dichroism in the $1 s \rightarrow \pi^{*}$ resonances indicate a flat adsorption geometry.

The carbon spectrum nicely resembles the naphthalene one [30,31], indicating no strong hybridization between the molecular and substrate electronic orbitals, as expected in the case of poor interaction. The boron K-edge presents several transitions in the 191-195 eV range of photon energy. The sharp peak marked by a green arrow in Figure 7 corresponds to the NEXAFS resonance of the aforementioned 2D framework of boroxine rings [29]. We tentatively ascribe it to the additional species related to the minor component we observe at $193 \mathrm{eV}$ in the B1s XPS. Peaks labeled 1, 2 and 3 in Figure 7 were assigned upon DFT simulation of the spectrum. Figure 8 shows the simulated total (unpolarized) spectrum. The main transitions are indicated, as well as the representation of the unoccupied molecular orbital involved in the corresponding transition. Peak 1 is related to the lowest unoccupied molecular orbital (LUMO), which is a $\pi^{*}$-symmetry orbital localized both on the B-O and B-C bonding regions; peaks 2 and 3 are associated with $\pi^{*}$ orbitals spatially extending to the whole naphthalene body. 


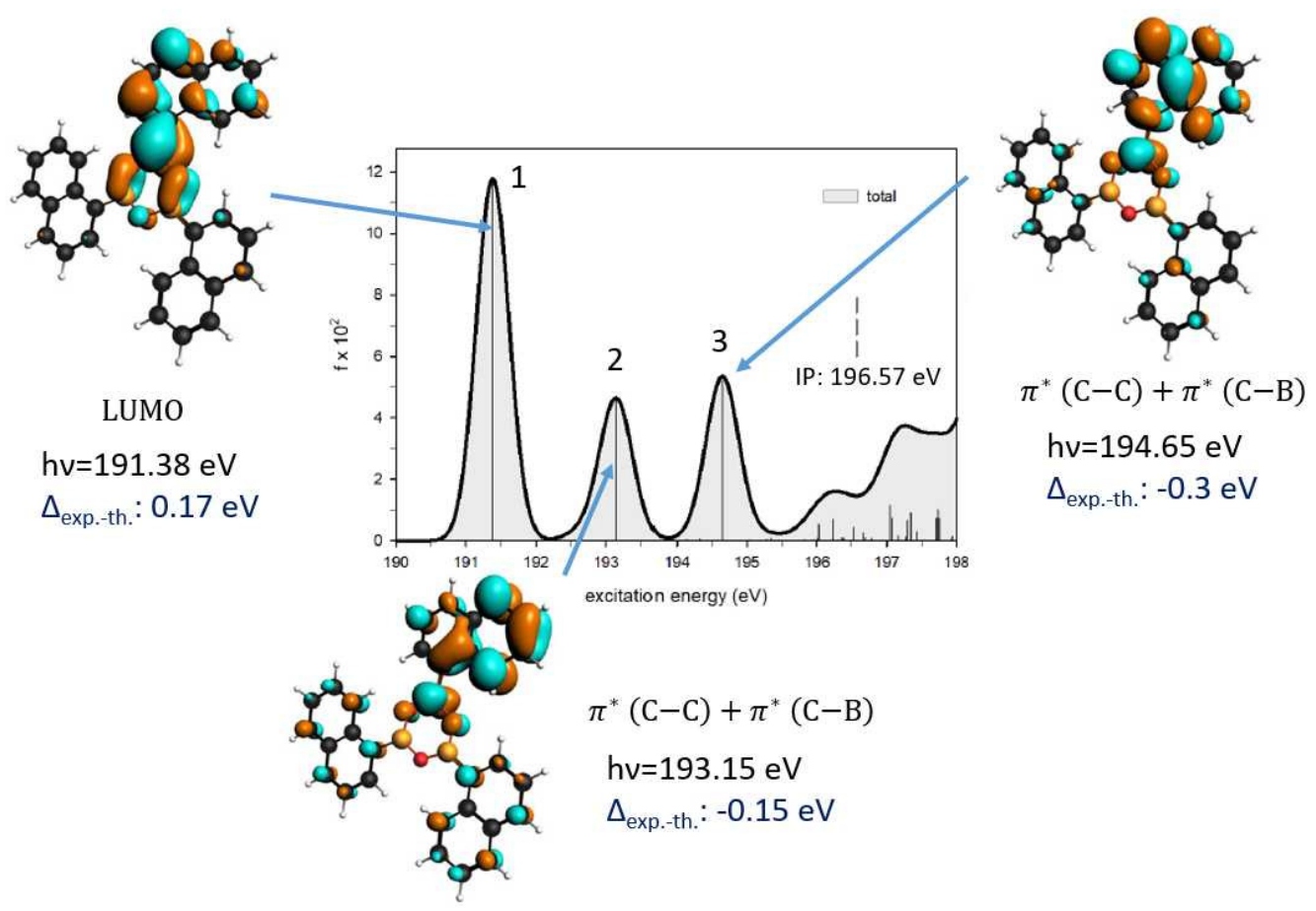

Figure 8. Calculated B K-edge unpolarized spectrum of the TNB molecule. The peaks originating from three main transitions, labeled 1, 2 and 3, correspond to the experimental resonances with the same numbering in Figure 7. For each peak, a representation of the molecular orbitals involved in the excitations is reported, together with the calculated photon energy position and its distance from the corresponding experimental value.

The positions of the calculated transitions are in fairly good agreement with the experimental values (see $\Delta_{\text {exp.th }}$. values reported in Figure 8); however, their relative intensities failed to match the experimental curve. This aspect was already studied in our previous research on triphenyl boroxine [16] and originates from static correlation effects, which should be taken into account [32]. The assignments of the molecular orbitals involved in the transitions are, however, reliable. They indicate that the first, most intense feature in the NEXAFS spectrum is mostly related to the boroxine ring of TNB, whereas other transitions may carry more information about the electronic coupling of boron atoms with the rest of the molecule.

\section{Conclusions}

The observed formation of TNB on $\mathrm{Au}(111)$ from NBA precursors proves that the synthesis method based on the on-surface boronic condensation can be extended to molecules other than the triphenyl boroxine, as initially reported. Our study opens the route to facile surface functionalization with macromolecules, whose chemical properties may be finely tuned by properly selecting boronic precursors. When adopting the boroxine structures as platforms for recognition of guest molecules, the NEXAFS resonances measured at the $B$ K-edge represent a convenient fingerprint of the boroxine chemical status. Observation of the different stages of the condensation process we performed by combining STM and XPS revealed an intermediate state, possibly characterized by the formation of covalent dimers. We propose a mechanism for the formation of the trimers, where two NBA molecules first condense in a dimer and then interact with a third molecule to complete the condensation of TNB. However, once a homogeneous phase of dimers is formed, the system does not evolve with the complete conversion into trimers upon thermal annealing. The kinetics of the reaction is likely dependent on the boronic precursor, as the dimer precursor stage was not observed in the case of triphenylboroxine formation. These findings could shed light 
on the on-surface boroxine formation mechanism upon further investigation and suggest novel strategies for the synthesis of boronic-derived structures.

Supplementary Materials: The following are available online at https:/ / www.mdpi.com/article/10 $.3390 /$ chemistry3040101/s1: discussion of the thermal treatment of LT and four-leaf phases and of the chirality of the molecular domains, with additional STM images.

Author Contributions: Conceptualization and supervision, A.C.; formal analysis, A.C., E.T., M.S., L.F., E.Z., D.T., G.F.; investigation, L.F., E.T., M.S., R.C., M.D., J.A.M., E.Z., D.T., G.F.; writing—original draft preparation, A.C., E.T., M.S.; writing—review and editing, all authors contributed to the revision of the manuscript; funding acquisition, A.M. All authors have read and agreed to the published version of the manuscript.

Funding: This research was partially funded by EUROFEL MIUR Progetti Internazionali. J.A.M. thanks to the TRIL programme of the ICTP.

Institutional Review Board Statement: Not applicable.

Informed Consent Statement: Not applicable.

Data Availability Statement: Data is contained within the article.

Conflicts of Interest: The authors declare no conflict of interest.

\section{References}

1. Bartels, L. Tailoring molecular layers at metal surfaces. Nat. Chem. 2010, 2, 87-95. [CrossRef]

2. Barth, J. V Molecular architectonic on metal surfaces. Annu. Rev. Phys. Chem. 2007, 58, 375-407. [CrossRef]

3. Di Giovannantonio, M.; Tomellini, M.; Lipton-Duffin, J.; Galeotti, G.; Ebrahimi, M.; Cossaro, A.; Verdini, A.; Kharche, N.; Meunier, V.; Vasseur, G.; et al. Mechanistic Picture and Kinetic Analysis of Surface-Confined Ullmann Polymerization. J. Am. Chem. Soc. 2016, 138, 16696-16702. [CrossRef]

4. Clair, S.; De Oteyza, D.G. Controlling a Chemical Coupling Reaction on a Surface: Tools and Strategies for On-Surface Synthesis. Chem. Rev. 2019, 119, 4717-4776. [CrossRef]

5. Grill, L.; Hecht, S. Covalent on-surface polymerization. Nat. Chem. 2020, 12, 115-130. [CrossRef]

6. Côté, A.P.; Benin, A.I.; Ockwig, N.W.; Matzger, A.J.; Yaghi, O.M. Porous, Crystalline, Covalent Organic Frameworks. J. Low Temp. Phys. 2004, 427, 155303. [CrossRef]

7. Waller, P.J.; Gándara, F.; Yaghi, O.M. Chemistry of Covalent Organic Frameworks. Acc. Chem. Res. 2015, 48, 3053-3063. [CrossRef]

8. Ma, L.; Wang, S.; Feng, X.; Wang, B. Recent advances of covalent organic frameworks in electronic and optical applications. Chinese Chem. Lett. 2016, 27, 1383-1394. [CrossRef]

9. Feng, X.; Ding, X.; Jiang, D. Covalent organic frameworks. Chem. Soc. Rev. 2012, 41, 6010. [CrossRef]

10. Zwaneveld, N.A.A.; Pawlak, R.; Abel, M.; Catalin, D.; Gigmes, D.; Bertin, D.; Porte, L. Organized formation of 2D extended covalent organic frameworks at surfaces. J. Am. Chem. Soc. 2008, 130, 6678-6679. [CrossRef]

11. Dienstmaier, F.; Gigler, A.M.; Goetz, A.J.; Knochel, P.; Bein, T.; Lyapin, A.; Reichlmaier, S.; Heckl, W.M.; Lackinger, M. Synthesis of Well-Ordered COF Monolayers: Surface Growth of Nanocrystalline Precursors versus Direct On-Surface Polycondensation. ACS Nano 2011, 5, 9737-9745. [CrossRef]

12. Dienstmaier, J.F.; Medina, D.D.; Dogru, M.; Knochel, P.; Bein, T.; Heckl, W.M.; Lackinger, M. Isoreticular two-dimensional covalent organic frameworks synthesized by on-surface condensation of diboronic acids. ACS Nano 2012, 6, 7234-7242. [CrossRef]

13. Colson, J.W.; Dichtel, W.R. Rationally synthesized two-dimensional polymers. Nat. Chem. 2013, 5, 453-465. [CrossRef]

14. Clair, S.; Abel, M.; Porte, L. Growth of boronic acid based two-dimensional covalent networks on a metal surface under ultrahigh vacuum. Chem. Commun. 2014, 50, 9627-9635. [CrossRef]

15. Plas, J.; Ivasenko, O.; Martsinovich, N.; Lackinger, M.; De Feyter, S. Nanopatterning of a covalent organic framework host-guest system. Chem. Commun. 2016, 52, 68-71. [CrossRef]

16. Toffoli, D.; Stredansky, M.; Feng, Z.; Balducci, G.; Furlan, S.; Stener, M.; Ustunel, H.; Cvetko, D.; Kladnik, G.; Morgante, A.; et al. Electronic properties of the boroxine-gold interface: Evidence of ultra-fast charge delocalization. Chem. Sci. 2017, 8, 3789-3798. [CrossRef]

17. Costantini, R.; Stredansky, M.; Cvetko, D.; Kladnik, G.; Verdini, A.; Sigalotti, P.; Cilento, F.; Salvador, F.; De Luisa, A.; Benedetti, D.; et al. ANCHOR-SUNDYN: A novel endstation for time resolved spectroscopy at the ALOISA beamline. J. Electron. Spectros. Relat. Phenomena 2018, 229, 7-12. [CrossRef]

18. Cossaro, A.; Floreano, L.; Verdini, A.; Casalis, L.; Morgante, A. Comment on “local methylthiolate adsorption geometry on $\mathrm{Au}(111)$ from photoemission core-level shifts". Phys. Rev. Lett. 2009, 103, 119601. [CrossRef]

19. Parr, R.G.; Yang, W. Density-Functional Theory of Atoms and Molecules; Oxford University Press: New York, NY, USA, 1989; ISBN 9780195092769.

20. Becke, A.D. Density-functional thermochemistry. III. The role of exact exchange. J. Chem. Phys. 1993, 98, 5648. [CrossRef] 
21. Lee, C.; Yang, W.; Parr, R. Development of the Colle- Salvetti correlation energy formula into a functional of the electron density. Phys. Rev. B 1988, 37, 785-789. [CrossRef]

22. Stephens, P.J.; Devlin, F.J.; Chabalowski, C.F.; Frisch, M.J. Ab Initio Calculation of Vibrational Absorption and Circular Dichroism Spectra Using Density Functional Force Fields. J. Phys. Chem. 1994, 98, 11623-11627. [CrossRef]

23. Triguero, L.; Pettersson, L.; Ågren, H. Calculations of near-edge X-ray-absorption spectra of gas-phase and chemisorbed molecules by means of density-functional and transition-potential theory. Phys. Rev. B-Condens. Matter Mater. Phys. 1998, 58, 8097-8110. [CrossRef]

24. Perdew, J.P. Density-functional approximation for the correlation energy of the inhomogeneous electron gas. Phys. Rev. B 1986, 33, 8822-8824. [CrossRef]

25. Baerends, E.J.; Ellis, D.E.; Ros, P. Self-consistent molecular Hartree-Fock—Slater calculations I. The computational procedure. Chem. Phys. 1973, 2, 41-51. [CrossRef]

26. Guerra, C.F.; Snijders, J.G.; te Velde, G.; Baerends, E.J. Towards an order-N DFT method. Theor. Chem. Acc. 1998, 99, $391-403$. [CrossRef]

27. Rettig, S.J.; Trotter, J. Crystal and molecular structure of phenylboronic acid, $\mathrm{C}_{6} \mathrm{H}_{5} \mathrm{~B}(\mathrm{OH})_{2}$. Can. J. Chem. 1977, 55, 3071-3075. [CrossRef]

28. Fournier, J.H.; Maris, T.; Wuest, J.D.; Guo, W.; Galoppini, E. Molecular tectonics. Use of the hydrogen bonding of boronic acids to direct supramolecular construction. J. Am. Chem. Soc. 2003, 125, 1002-1006. [CrossRef]

29. Stredansky, M.; Sala, A.; Fontanot, T.; Costantini, R.; Africh, C.; Comelli, G.; Floreano, L.; Morgante, A.; Cossaro, A. On-surface synthesis of a 2D boroxine framework: A route to a novel 2D material? Chem. Commun. 2018, 54, 3971-3973. [CrossRef]

30. Minkov, I.; Gel'mukhanov, F.; Friedlein, R.; Osikowicz, W.; Suess, C.; Öhrwall, G.; Sorensen, S.L.; Braun, S.; Murdey, R.; Salaneck, W.R.; et al. Core excitations of naphthalene: Vibrational structure versus chemical shifts. J. Chem. Phys. 2004, 121, 5733-5739. [CrossRef]

31. Dri, C.; Fronzoni, G.; Balducci, G.; Furlan, S.; Stener, M.; Feng, Z.; Comelli, G.; Castellarin-Cudia, C.; Cvetko, D.; Kladnik, G.; et al. Chemistry of the Methylamine Termination at a Gold Surface: From Autorecognition to Condensation. J. Phys. Chem. C 2016, 120, 6104-6115. [CrossRef]

32. Toffoli, D.; Ponzi, A.; Bernes, E.; De Simone, M.; Grazioli, C.; Coreno, M.; Stredansky, M.; Cossaro, A.; Fronzoni, G. Correlation effects in B1s core-excited states of boronic-acid derivatives: An experimental and computational study. J. Chem. Phys. 2019, 151, 134306. [CrossRef] 